\title{
Modeling environmental risk factors of autism in mice induces IBD-related gut microbial dysbiosis and hyperserotonemia
}

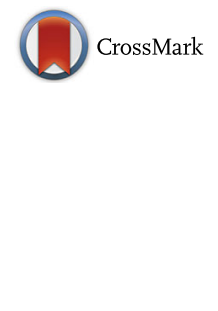

\author{
Joon Seo Lim ${ }^{1,2+}$, Mi Young Lim ${ }^{1,2,3+}$, Yongbin Choi ${ }^{1}$ and GwangPyo Ko ${ }^{1,4,5,6^{*}}$
}

\begin{abstract}
Autism spectrum disorder (ASD) is a range of neurodevelopmental conditions that are sharply increasing in prevalence worldwide. Intriguingly, ASD is often accompanied by an array of systemic aberrations including (1) increased serotonin, (2) various modes of gastrointestinal disorders, and (3) inflammatory bowel disease (IBD), albeit the underlying cause for such comorbidities remains uncertain. Also, accumulating number of studies report that the gut microbial composition is significantly altered in children with ASD or patients with IBD. Surprisingly, when we analyzed the gut microbiota of poly I:C and VPA-induced mouse models of ASD, we found a distinct pattern of microbial dysbiosis that highly recapitulated those reported in clinical cases of ASD and IBD. Moreover, we report that such microbial dysbiosis led to notable perturbations in microbial metabolic pathways that are known to negatively affect the host, especially with regards to the pathogenesis of ASD and IBD. Lastly, we found that serum level of serotonin is significantly increased in both poly I:C and VPA mice, and that it correlates with increases of a bacterial genus and a metabolic pathway that are implicated in stimulation of host serotonin production. Our results using animal model identify prenatal environmental risk factors of autism as possible causative agents of IBD-related gut microbial dysbiosis in ASD, and suggest a multifaceted role of gut microbiota in the systemic pathogenesis of ASD and hyperserotonemia.
\end{abstract}

\section{Introduction}

Autism spectrum disorder (ASD) is characterized by core deficits in neurodevelopmental milestones. However, a majority of children with ASD also suffer from a wide range of systemic aberrations such as increased serum serotonin (40\% prevalence) [1-3], gastrointestinal (GI) distress (up to 90\%) [4], and inflammatory bowel disease (IBD) [5]. Unfortunately, the causative agent for those conditions and their relationship with etiology and pathogenesis of ASD remains undefined.

Gut microbiota carries a central role in the health status of its host via regulation of immune system and metabolism [6]. Intuitively, one of the most directly affected host site by microbial dysbiosis is the GI tract,

\footnotetext{
* Correspondence: gko@snu.ac.kr

${ }^{\dagger}$ Equal contributors

'Department of Environmental Health Sciences, Graduate School of Public Health, Seoul National University, Seoul, Republic of Korea

${ }^{4}$ Center for Human and Environmental Microbiome, Seoul National

University, Seoul, Republic of Korea

Full list of author information is available at the end of the article
}

in that dysbiosis is responsible for the development of GI disorders and IBD [7]. Gut microbiota is also able to affect the host's neurodevelopmental status via gutbrain-axis [8]. Importantly, a recent paper demonstrated that administration of beneficial bacteria can ameliorate a subset of behavioral abnormalities in a mouse model of autism [9]. Moreover, gut microbiota profoundly impacts host serotonin production [10], an imbalance of which leads to GI discomfort and altered mental status [11].

Co-occurrence of two or more mutually exclusive, etiologically unrelated diseases is rare; therefore, the fact that GI disorders and IBD are so prevalent among children with ASD is highly suggestive of a common cause for the behavioral abnormalities and systemic aberrations in ASD. Accordingly, accumulating number of studies report that the gut microbial composition and several microbial metabolic pathways are significantly altered in children with ASD $[12,13]$ as well as patients with IBD $[14,15]$. 
The rising global incidence of ASD [16] implies that environmental factors might be a contributing component in its etiology. We therefore hypothesized that environmental risk factors are responsible for the gut microbial dysbiosis in ASD, and that such dysbiosis is a driving force for the wide range of systemic aberrations in ASD. To test this, we chose prenatal injection of polyinosinic:polycytidylic acid (poly I:C) and valproic acid (VPA) as our model, which represent two of the most widely utilized environmental risk factors of ASD. We analyzed the overall composition of gut microbiota in poly I:C and VPA mice as well as the relative abundances of 259 microbial taxa and compared the results to existing reports on microbial dysbiosis in clinical cases of ASD and IBD. We also performed network analysis to examine the correlation between microbial families, and applied the relative abundance data to metabolic pathway database in order to assess how microbial dysbiosis might affect the host's metabolic system in ASD. Lastly, we tested if ASD mice also display hyperserotonemia, another highly occurring comorbidities in ASD.

\section{Methods \\ Generation of environmental risk factor-induced mouse models of autism}

Pregnant C57BL/6 mice from Japan SLC, Inc. (Japan) were purchased via Central Lab. Animal Inc. (Seoul, Korea) and housed in animal biosafety level 2 SPF facility at Seoul National University College of Medicine, Korea, with ad libitum supply of standard chow and water. At embryonic day 12.5 (E12.5), a total of 15 pregnant dams were intraperitoneally injected with poly I:C (20 mg/kg; Sigma-Aldrich, USA), valproic acid (VPA) $(500 \mathrm{mg} / \mathrm{kg}$; Sigma-Aldrich, USA), or equivalent amount of saline (5 dams per group). The pups were weaned at three weeks after birth and were supplied with the same kind of chow and water. Only male pups were used for all subsequent experiments.

\section{Behavioral assay}

Poly I:C and VPA offspring were subjected to behavioral assays relevant to autism phenotype at six weeks of age. The mice were first tested for anxiety-related behavior using open field chamber $(42 * 42 * 42 \mathrm{~cm})$, in which exploratory behavior in a novel environment was assessed in 10-minute sessions. Locomotor activity was captured with a video camera and analyzed with Ethovision XT software (Noldus, USA). The amount of total movement during the experimental session as well as time spent in the center region $(30 * 30 \mathrm{~cm}$ area) was measured and compared to corresponding control (CTL) group.
The mice were also tested for social interaction behavior using 3-chamber assay [9]. Briefly, testing mice were habituated for $10 \mathrm{~min}$ in the chamber; then two cylindrical cages were placed on each side of the chamber, one of which contained a stranger (mouse of the same sex and age raised in a separate cage), thus testing for social preference over the non-social cue. $10 \mathrm{~min}$ after the initial interaction, a second stranger mouse was placed in the empty cage and the behavior of the testing mouse was again recorded for $10 \mathrm{~min}$, which represents a preference for a novel social cue. The relative time spent on each cylindrical cage during each experimental phase was calculated and compared to that of CTL mice.

\section{Serum serotonin measurement}

Blood samples were collected from submandibular veins of mother and offspring mice by using Goldenrod Animal Lancet (Braintree Scientific, Inc., USA) according to the manufacturer's instructions. Serum was separated from whole blood by allowing the blood to clot at room temperature, then centrifuging at $1,000 \mathrm{x}$ g for 10 minutes at $4{ }^{\circ} \mathrm{C}$. The resulting serum supernatant was then stored in $-20{ }^{\circ} \mathrm{C}$ until use. Serum levels of serotonin were measured using commercial ELISA assay (Eagle Biosciences, USA) according to the manufacturer's instructions, and values from poly I:C and VPA groups were compared to the corresponding CTL groups of the same age in both mother and offspring mice.

\section{DNA extraction and Illumina sequencing of mouse fecal DNA}

Fecal samples from mother and offspring mice were collected at indicated time points and immediately placed in liquid nitrogen. DNA was extracted from feces using QIAamp DNA Stool Mini Kit according to the manufacturer's instruction (Qiagen, USA), and stored in $-20{ }^{\circ} \mathrm{C}$ until use. The fecal DNA samples were PCRamplified using Illumina-adapted universal primers $515 \mathrm{~F}$ and $806 \mathrm{R}$ that target the V4 region of the $16 \mathrm{~S}$ rRNA gene [17], and the amplicons were quantified with the KAPA Library Quantification Kit (KAPA Biosystems, USA). The resulting amplicons from each sample were sequenced using the MiSeq platform (Illumina, USA), which yielded $16 \mathrm{~S}$ rRNA data for further analysis.

\section{$16 \mathrm{~S}$ rRNA gene sequence processing and statistical analysis}

$16 \mathrm{~S}$ data from mice fecal DNA were processed and analyzed using QIIME 1.8 software package [18]. First, the sequences were clustered into operational taxonomic units (OTUs) at 97\% identity using OTU-picking protocol, and the relative abundances of microbial taxa from genus to kingdom were generated from nonrarefied OTU table. Alpha-diversity indexes (PD index, 
Shannon diversity index) were estimated and tested for significant differences between CTL and poly I:C or VPA groups using Monte Carlo permutations. Betadiversity using unweighted UniFrac distance was calculated onto a rarefied OTU table, which was grouped into either timing of sampling or drug treatment.

To evaluate the co-occurrence relationships between microbial taxa, we calculated Spearman's ranked correlation of their relative abundances, and the networks of co-occurring taxa were then visualized using Cytoscape $[19,20]$. Each node represents a microbial family (e.g., Prevotellaceae) and the nodes were paired when their $\mathrm{q}$-values and $\mathrm{p}$-values were both below 0.05 , respectively. The length of the linkage is proportional to the closeness of the two families. Microbial functions from the $16 \mathrm{~S}$ data were predicted by using PICRUSt [21] and collapsing the predicted functions into higher categories according to Kyoto Encyclopedia of Genes and Genomes (KEGG) orthology as previously described [22], and the resulting functioning profiles were visualized as a heatmap using $R$ package [23].

Statistical significance of differences between CTL and poly I:C or VPA groups in each experiment were calculated using either R or Microsoft Excel 2013 using paired t-test. Differences between groups were determined significant at $" p<0.05,{ }^{* * *} p<0.01,{ }^{* * * *} p<0.001$, and ${ }^{* * * * * *} p<0.0001$.

\section{Results}

\section{ASD mice have reduced gut bacterial diversity}

According to previous literature on generating environmental risk factor-induced ASD model, we intraperitoneally injected pregnant C57BL/6 mice with $20 \mathrm{mg} / \mathrm{kg}$ poly I:C [9, 24] or $500 \mathrm{mg} / \mathrm{kg}$ VPA [25] at embryonic day 12.5 (E12.5). When subjected to a battery of standard behavioral testing at six weeks of age, both poly I:C and VPA offspring, hereafter referred to as ASD mice, showed autism-related behavioral abnormalities including increased anxiety and decreased social behavior (Additional file 1: Figure S1).

We analyzed the composition of fecal microbiota of post-weaned postnatal day 21 (P21) mice, and observed that the overall composition of gut microbes of ASD mice did not significantly differ from that of CTL mice at the phylum level, except for a slight increase in Firmicutes accompanied by a roughly equal amount of decrease in Bacteroidetes (Fig. 1a). On the other hand, analysis of two alpha-diversity indexes (PD index and Shannon index) showed that ASD mice harbor significantly less diverse microbial species (Figs. 1b and c), which is in line with previous findings in children with ASD [13].
Dietary change, but not poly I:C or VPA, induces betadiversity shifts in mice

Beta-diversity index measures the compositional difference of microbial community between experimental groups [26], and denotes if a general shift in the microbial community occurred in response to a stimulus, which in this case was ASD induction. We analyzed fecal samples from CTL and ASD mice right before weaning at P21, and twice more in one-week intervals (P28, P35). Unweighted betadiversity analysis showed that as the mice went through dietary change from mother's milk (P21) to standard chow (P28, P35), their gut microbiota experienced a distinguishable shift in their composition (Fig. 1d). However, in each of those time points, the microbial communities of ASD mice were not clearly distinguished from that of CTL mice (Fig. 1e). Collectively, the gut microbiota of ASD mice was not distinguishable from that of CTL mice at the phylum level or in their general composition; rather, these results suggest that the decrease in their alpha-diversity indexes might stem from dysbiosis at the genus or species level.

\section{ASD- and IBD-related gut bacterial species are altered in ASD mice}

A number of studies have reported that children with ASD $[12,13,27-29]$ or patients with IBD [15, 30-39] have altered gut microbial composition at the genus or species level, some of which are consistently reported across a number of clinical studies (summarized in Table 1).We therefore performed relative abundance analysis of gut microbiota of ASD mice at various taxonomic levels ranging from phylum to species, and found that prenatal injection of poly $\mathrm{I}: \mathrm{C}$ and VPA resulted in significant changes in specific microbial taxa in a pattern that highly recapitulates those of clinical ASD and IBD (Fig. 2). Both poly I:C and VPA mice showed significant increases in bacterial species that are abundant in ASD (Fig. 2a) or IBD (Fig. 2b), including Enterococcus [40], which has been suspected to have a causal relationship with the disease progression of IBD. ASD mice also had significantly less abundant species that are decreased in clinical IBD (Fig. 2c), and in the case of Desulfovibrio, both the children with ASD and Crohn's disease patients had increased abundance of the genus, a pattern also observed in ASD mice (Fig. 2d). Lastly, ASD mice had decreased abundance of Oscillospira sp., F. Prausnitzii, and Prevotella-all of which are underrepresented in clinical cases of ASD and IBD (Fig. 2d). Comprehensive lists of microbial taxa significantly altered in poly I:C and VPA mice are provided in the online version of this manuscript (Additional file 4: Table S1).

\section{Prevotellaceae family is significantly and independently decreased in ASD mice}

Out of the 259 microbial taxa tested, the genus Prevotella showed the most prominent change compared to CTL, 


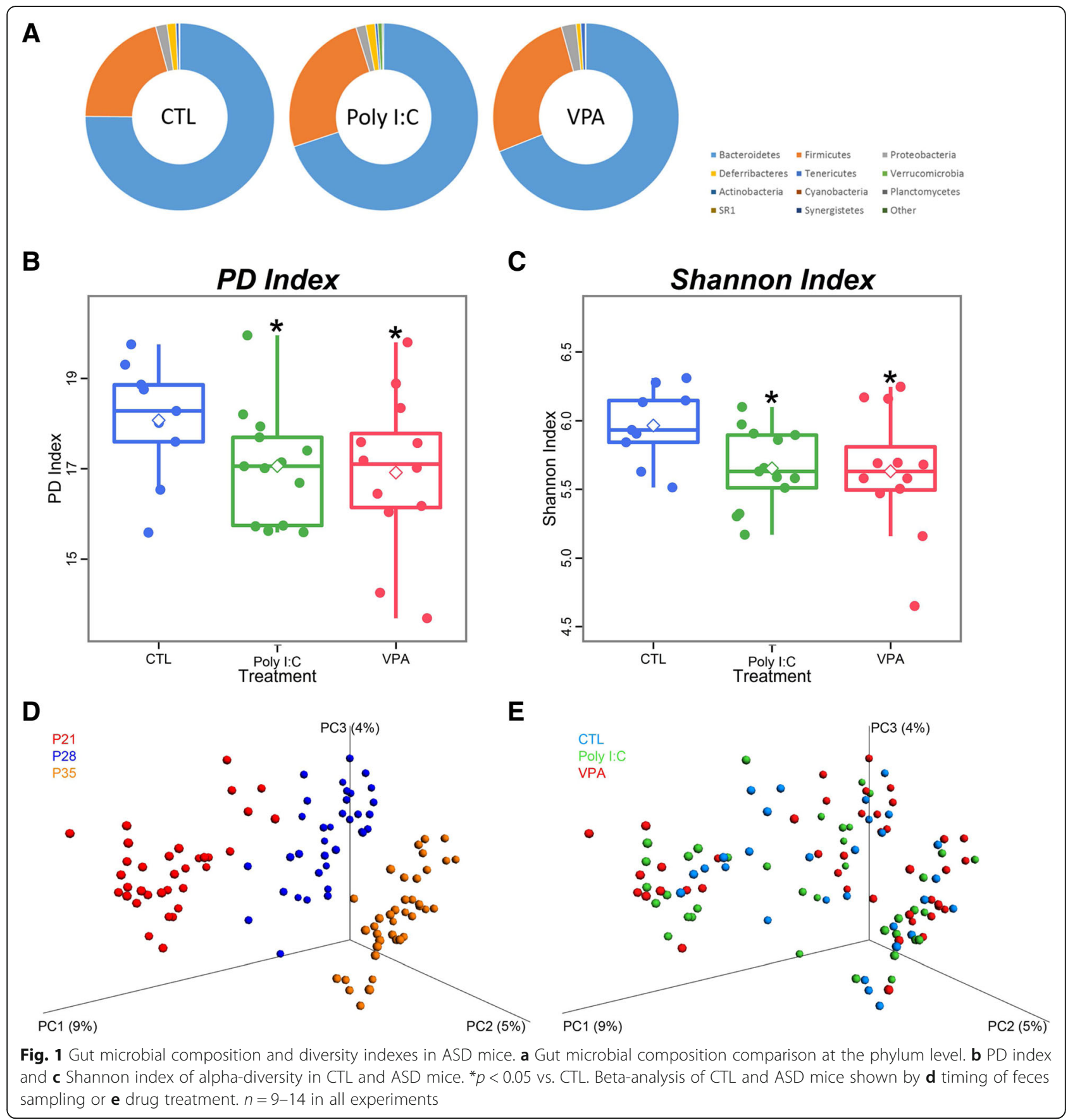

with statistical significance well below $p<0.0001$ (Fig. 2d) in both poly I:C and VPA mice. When we reanalyzed the gut microbial abundance at the family level, Prevotellaceae comprised more than $7 \%$ of the total gut microbial community in CTL mice, whereas it only spanned $\sim 2.5 \%$ in ASD mice, with many specimens showing close to zero percentage abundances (Fig. 3a). We then performed co-occurrence analysis based on Spearman's rank correlations to study how microbial families were interlinked (Fig. 3b), and found that families such as Rikenellaceae, Peptoccaceae, and Lactobacillaceae were significantly correlated to more than dozen other families, indicating that as the abundances of those families change, so do the abundances of other dozen families. However, Prevotellaceae was negatively correlated to only four other families-Rikenellaceae, Lachnospiraceae, Anaeroplasmataceae, and Peptostreptococcaceae; moreover, when we applied weight to the co-occurrence data so that closely linked families will be shown as such, Prevotellaceae was 
Table 1 Bacterial taxa reported as altered in clinical cases of ASD and IBD

\begin{tabular}{llll}
\hline & Bacteria & vs. HC & Reference \\
\hline ASD-related taxa & Dorea sp. & $\uparrow$ & {$[12]$} \\
& Parabacteroides & $\uparrow$ & {$[28]$} \\
& Desulfovibrio & $\uparrow$ & {$[28]$} \\
& Oscillospira sp. & $\downarrow$ & {$[12]$} \\
& F. Prausnitzii & $\downarrow$ & {$[12]$} \\
& Prevotella & $\downarrow$ & {$[12][13]$,} \\
IBD-related taxa & Bilophila & $\uparrow$ & {$[30]$} \\
& Enterococcus & $\uparrow$ & {$[32]$} \\
& Megasphaera & $\uparrow^{* * *}$ & {$[33]$} \\
& Bacteroides ovatus & $\uparrow$ & {$[34]$} \\
& Peptostreptococcaceae & $\uparrow^{* *}$ & {$[35]$} \\
& Coriobacteriaceae & $\downarrow^{* * * *}$ & {$[50]$} \\
& P. distasonis & $\downarrow$ & {$[78]$} \\
& Erysipelotrichaceae & $\downarrow^{*}$ & {$[36]$} \\
& Desulfovibrio & $\uparrow^{* *}$ & {$[37]$} \\
& Oscillospira sp. & $\downarrow^{*}$ & {$[38]$} \\
& F. prausnitzii & $\downarrow^{*}$ & {$[79]$} \\
& Prevotella & $\downarrow$ & {$[15]$}
\end{tabular}

Bold taxa are reported to be altered in both ASD and IBD. The asterisks $\left({ }^{*}, * *\right.$, and $\left.{ }^{* * *}\right)$ next to the arrows denote alterations reported in Crohn's Disease, ulcerative colitis, and irritable bowel syndrome, respectively

shown to be separated from all the other families by a great margin (Fig. 3c). This result suggests that dysbiosis in other families is not likely to have caused a noticeable shift in the abundance of Prevotellaceae, and vice versa. Taken together, the decrease in Prevotella, which has been reported in clinical cases of both ASD and IBD, is highly unlikely to have been brought forth by alterations in other microbial communities in our mouse model, but more likely by an inhospitable host environment due to ASD induction.

\section{Metabolic pathways implicated in ASD and IBD are altered in KEGG pathway analysis of poly I:C and VPA mice}

We have thus far examined how ASD induction by environmental risk factors leads to clinically-relevant microbial dysbiosis in the offspring. Consequently, we sought to get an insight of how microbial dysbiosis in turn affects the metabolism of ASD mice. We assessed the functional potential of each experimental groups by applying the 16S data into Kyoto Encyclopedia of Genes and Genomes (KEGG) pathway abundances [22]. As a result, we found that many pathways that have previously been implicated in ASD (dioxin degradation [41], steroid hormone biosynthesis [42]) were altered in the same manner in ASD mice (Fig. 4a, Table 2), as well as those implicated in IBD (sulfur metabolism [43], N-Glycan biosynthesis; [44] Table 2). Notably, lipopolysaccharide
(LPS) biosynthesis and bacterial toxin have been implicated in the pathogenesis of both ASD [45] and IBD; [46] accordingly, our results showed that both poly I:C and VPA mice have significant up-regulations in pathways involving LPS biosynthesis and bacterial toxins (Fig. 4a). Comprehensive lists of metabolic pathways significantly altered in poly I:C and VPA mice are provided in the online version of this manuscript (Additional file 5: Table S2).

\section{ASD mice have elevated level of serum serotonin}

Interestingly, we observed one pathway that was upregulated in VPA $(p=0.047)$ and poly I:C mice $(p=$ $0.055)$, which was not directly implicated in the disease progression of either ASD or IBD: "Protein digestion and absorption (hsa04974)". Nevertheless, this pathway was of potential interest in that it is implicated in the production of short chain fatty acids (SCFAs), which are known to stimulate serotonin production from enterochromaffin cells [47]. Elevated serum serotonin, along with GI disorders and IBD, is another highly occurring comorbidity in ASD [3]. Importantly, a recent publication has shown that the gut microbes are responsible for stimulating host serotonin production, and noted that spore-forming bacteria of Clostridia class are responsible for such action.

We therefore analyzed the relative abundance of Clostridia class bacteria in ASD mice and found that Clostridiales Tissierellaceae Sporanaerobacter, a genus that forms spores and produces SCFAs [48], was significantly increased in both poly I:C and VPA mice (Fig. 4b). The increase in SCFA-producing pathway in KEGG analysis, as well as the increased abundance of spore-forming, aceticacid producing bacteria Sporanaerobacter, indicated that serum serotonin might be increased in these mice as well. Indeed, when we analyzed the serum from ASD mice, we observed a significant increase of serum serotonin level compared to CTL mice (Fig. 4c), a phenomenon not previously reported in poly I:C and VPA mice models of ASD.

Taken together, our results show that microbial dysbiosis induced by prenatal poly I:C and VPA treatment not only recapitulated many aspects of microbial dysbiosis in clinical ASD and IBD, but may also have functional properties that affect the host's health status via alterations in metabolism and serotonin production.

\section{Discussion}

Autism spectrum disorder (ASD) now affects approximately 1 in 161 children globally [16], and is continuing to rise in prevalence especially in developed countries. Moreover, children with ASD are commonly burdened with various gastrointestinal (GI) disorders [4] ranging from mild GI discomfort and constipation [49] to inflammatory bowel disease (IBD). Numerous studies have also reported significant alterations in the composition of gut microbes in ASD children burdened with GI 


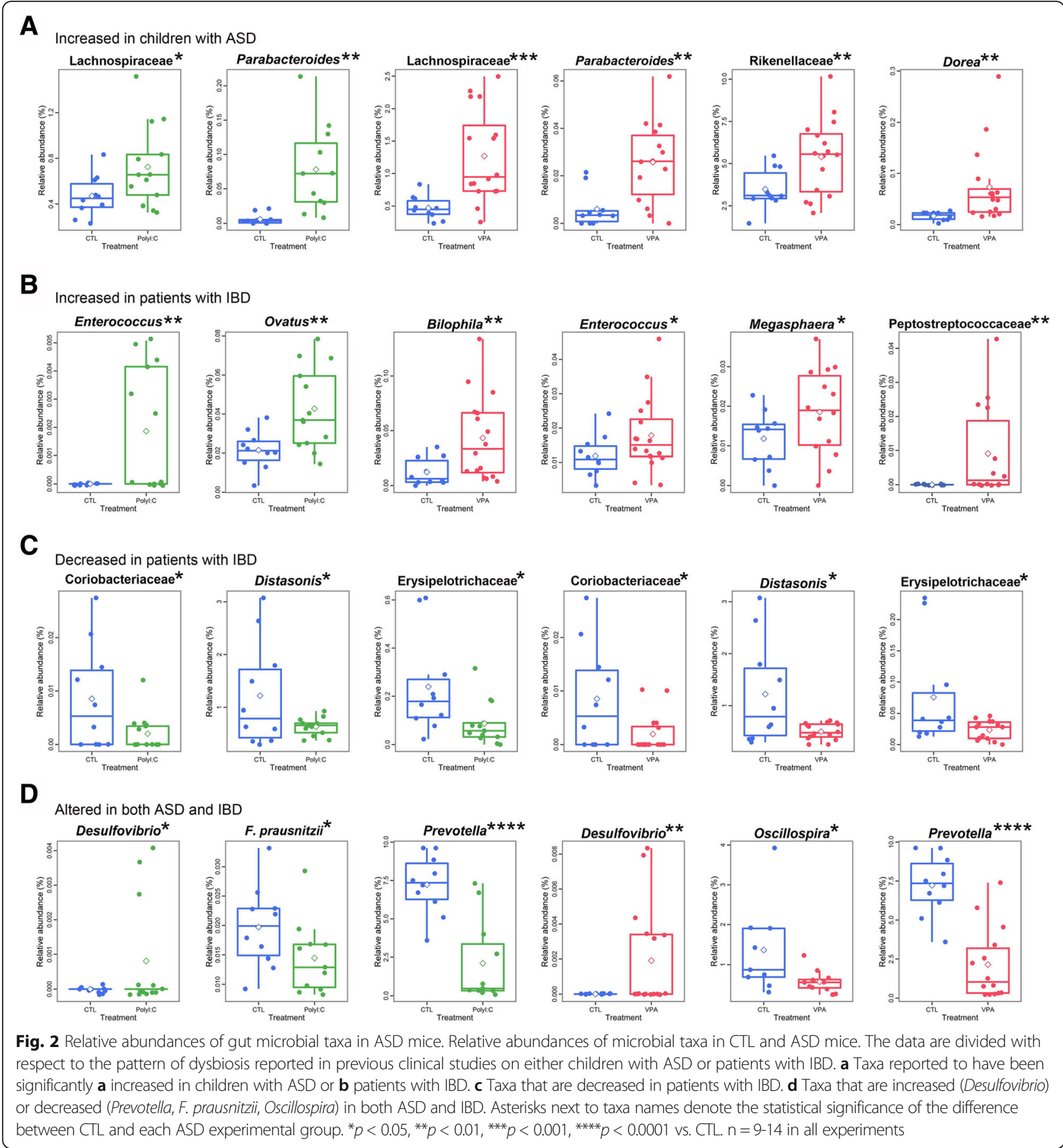

disorders $[12,13,28,29]$, the dysbiosis of which is highly responsible for the development of GI disorders and IBD [7]. Approximately $40 \%$ of children with ASD also have elevated level of serotonin [2,3], which might cause diarrhea and even altered mental status [11]. In this animal model study, we showed that modeling two environmental risk factors of autism - prenatal injection of poly $\mathrm{I}: \mathrm{C}$ or valproic acid (VPA) - results in a distinct pattern of gut microbial dysbiosis that highly recapitulates those observed in children with ASD or patients with IBD (Fig. 2). We have also observed that ASD mice have gut microbial dysbiosis with decreased alpha-diversity compared to CTL mice, which is in line with few previous publications on gut microbial profiling of children with ASD [13] or patients with IBD [14]. This suggests that prenatal events could act as causal agents for microbial alteration responsible for IBD $[50,51]$, one of the most common comorbidities observed in ASD. 


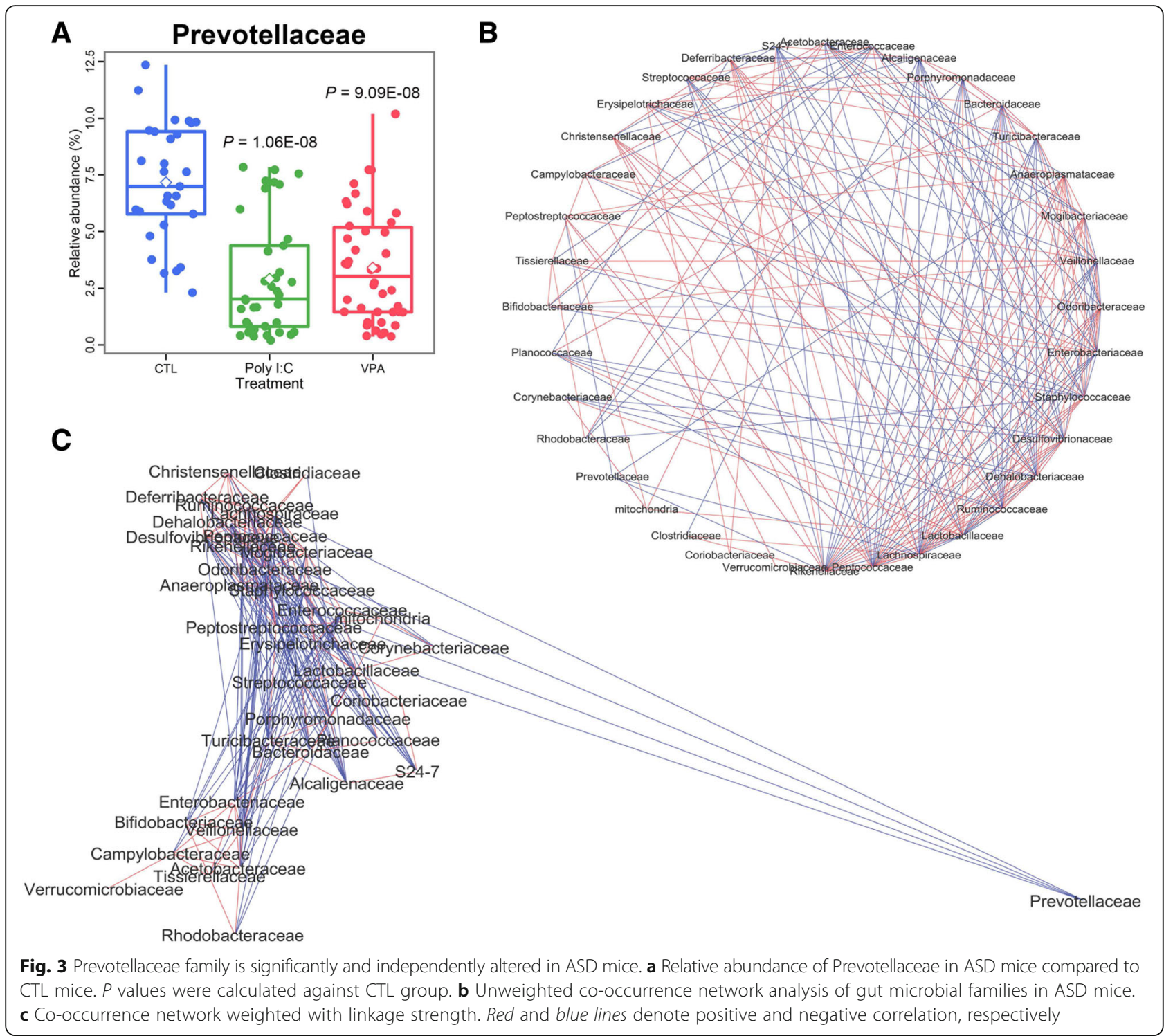

Our result on IBD-like microbial dysbiosis in two environmental risk factor models of ASD adds support to previous studies that have investigated how prenatal injection of poly I:C or VPA affects the GI tract in offspring later in life. Similar to patients with IBD [52], poly I:C offspring exhibit GI barrier defects such as increased permeability, and abnormal expression of tight junction proteins in the gut [9]. In addition, poly I:C offspring display significantly elevated level of plasma cytokines such as IL-2, IL-5, and IL-6 [53]. As for VPA, male VPA offspring have been shown to display epithelial loss in the ileum as well as signs of neutrophil infiltration in their gut [54], a phenomenon frequently observed in clinical cases of IBD $[55,56]$. Prenatal VPA injection in rats resulted in reduced thickness of the mucosa and muscle layers of GI tract in the offspring, which was accompanied by significant changes in the morphologies of GI epithelial cells such as atrophy, weak cytoplasm staining, and distracted arrangement of chief cells; moreover, the GI transit index was significantly decreased in the VPA offspring, indicating functional deficit of GI in response to prenatal VPA injection [57]. Taken together, previous studies have shown that prenatal administration of environmental risk factors of ASD can induce IBD-like symptoms in the offspring, and thereby suggest possible connection between ASD and IBD at the tissue level.

Out of the 259 taxa tested, the most prominently and uniquely altered taxa was Prevotella, a fermenter of plant polysaccharides. For this reason, it has been suggested that decrease in Prevotella in the intestine might be associated with various digestive disorders [13]. Accordingly, clinical reports have shown that Prevotella is significantly decreased in both autistic individuals [13] 


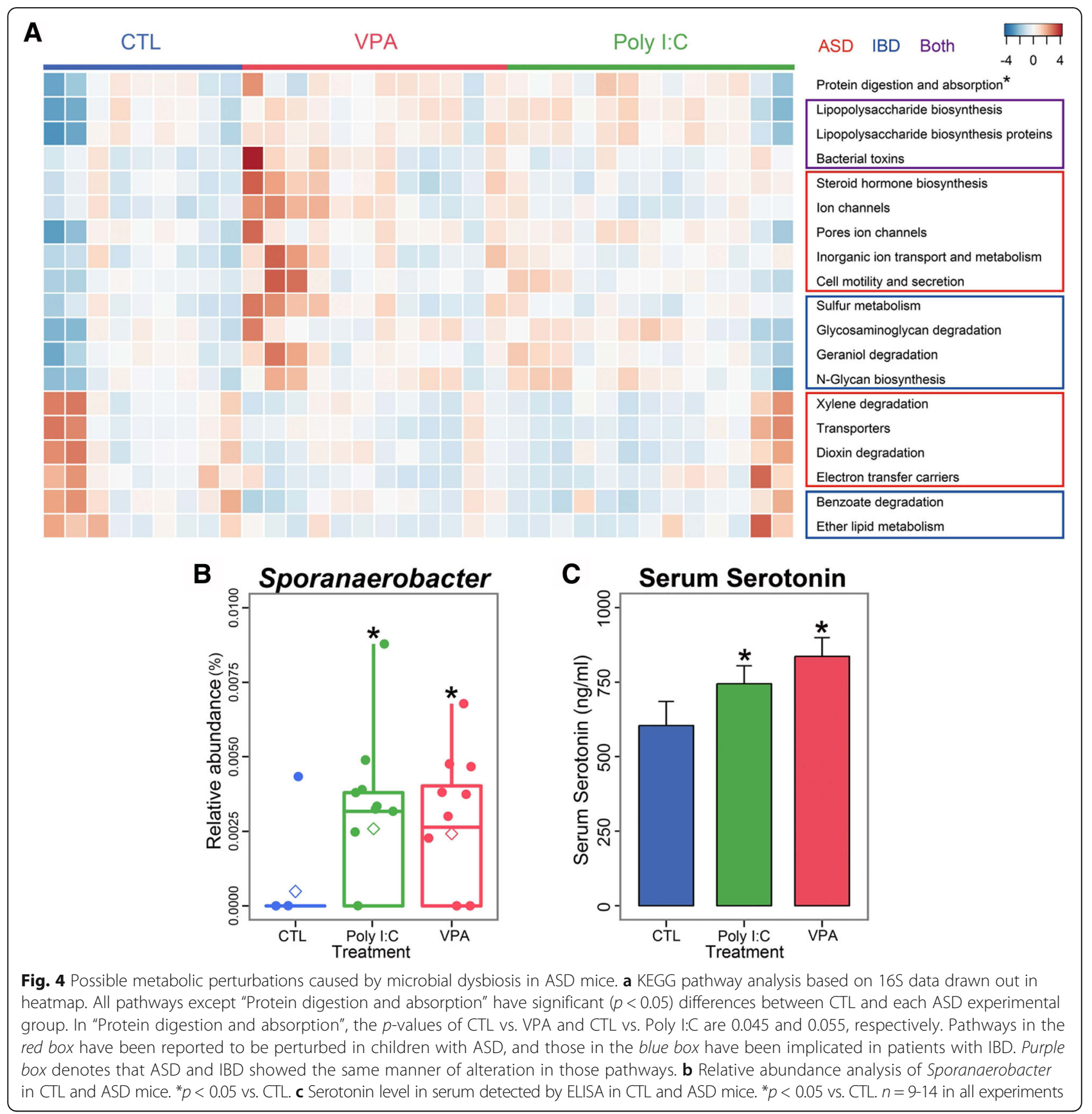

and IBD patients [15], indicating that changes in the relative abundance of Prevotella might be a key factor that links ASD and IBD. Prevotella has also recently attracted attention due to its distinct pattern of abundance in response to diet, in that it is highly prevalent in rural Africa and significantly reduced in the Western world [58], the latter of which harbors countries with one of the most highest incidence of ASD [16]. Notably, our present results bring a new insight to the relationship between ASD and Prevotella, in that prenatal environmental risk factors of ASD may render the host's enteric environment inhospitable for the growth of Prevotella, an effect which seems to have overcome the controlled dietary environment in our study setting. It would be of potential value to test if colonization of Prevotella or administration of prebiotics aimed at increasing its prevalence might alter the inflammatory status of ASD mice or even its behavioral abnormalities.

We also showed that microbial dysbiosis is likely to affect the host's health status via alteration of its metabolic pathways (Fig. 4a). A variety of metabolic disturbances has been reported in ASD [41, 42, 59-65] and 
Table 2 Metabolic pathways implicated in clinical cases of ASD and IBD

\begin{tabular}{llll}
\hline & Pathway & vs. HC & Reference \\
\hline ASD-related & LPS/LPS proteins/Bacterial toxins & $\uparrow$ & {$[45]$} \\
pathways & Steroid hormone biosynthesis & $\uparrow$ & {$[42]$} \\
& Ion channels/Pores ion channels & $\uparrow$ & {$[59]$} \\
& Inorganic ion transport and & $\uparrow$ & {$[60]$} \\
& metabolism & & \\
& Cell motility and secretion & $\uparrow$ & {$[61]$} \\
& Xylene degradation & $\downarrow$ & {$[63]$} \\
& Transporters & $\downarrow$ & {$[64]$} \\
& Dioxin degradation & $\downarrow$ & {$[41]$} \\
& Electron transfer carriers & $\downarrow$ & {$[65]$} \\
IBD-related & LPS/LPS proteins/Bacterial toxins & $\uparrow$ & {$[46]$} \\
pathways & Sulfur metabolism & $\uparrow$ & {$[43]$} \\
& Glycosaminoglycan degradation & $\uparrow$ & {$[67]$} \\
& Geraniol degradation & $\uparrow$ & {$[68]$} \\
& N-Glycan biosynthesis & $\uparrow$ & {$[44]$} \\
& Benzoate degradation & $\downarrow$ & {$[51]$} \\
& Ether lipid metabolism & $\downarrow$ & {$[69]$} \\
\hline
\end{tabular}

Bold pathways are reported to be altered in both ASD and IBD

IBD [43, 51, 66-69], with a possible relationship to the etiology or progression of those conditions. One example is the elevated level of lipopolysaccharide (LPS) and bacterial endotoxins: in ASD, an elevation of circulating level of bacterial endotoxin in ASD patients compared to healthy controls (HCs) was reported [45], and it was repeatedly demonstrated in murine models that early life exposure to LPS results in ASD-like behavioral abnormalities via inflammatory responses [70, 71]. With regards to IBD, endotoxemia was also reported in a major subset of patients with ulcerative colitis or Crohn's disease [46], as well as serum markers of LPS exposure in pediatric IBD patients [66]. Interestingly, ASD mice also showed an elevated level of metabolic pathways associated with LPS: "Lipopolysaccharide biosynthesis", "Lipopolysaccharide biosynthesis proteins", and "Bacterial toxins" (Fig. 4a, purple box). Accordingly, one research group hypothesized that toxin-producing harmful gut bacterial species such as Desulfovibrio might contribute to ASD [29], and later reported that Desulfovibrio was found more frequently in the stools of autistic children compared to HCs [28]. Similarly, we also observed that the relative abundance of Desulfovibrio is significantly increased in both poly I:C and VPA mice (Fig. 2d). Taken together, our results link poly I:C- and VPA-induced microbial dysbiosis with endotoxemia implicated in both ASD and IBD, thereby lending more support to the microbial basis of ASD etiology and pathogenesis.

Hyperserotonemia in autistic individuals was first reported more than fifty years ago [72], and has been continued to be described in major subsets of autistic children to the point that researchers have suggested elevated serum serotonin as a possible cause or aggravator of ASD-like behavioral phenotypes [1,3]. One study also proposed that serotonin pathway can be utilized as a biomarker for autism diagnosis [2], although the underlying cause for such aberration remained unclear. Recently, expression of a variant in serotonin transport gene (SLC6A4) in mice was shown to result in hyperserotonemia and ASD-related behavioral abnormalities [73]. However, no single genetic variant or mutation related to ASD accounts for more than 1-2\% of the total affected population [74], a figure falling far short of the prevalence of hyperserotonemia (40\%). Instead, we here report for the first time that modeling environmental risk factors of ASD in mice resulted in a significantly elevated level of serum serotonin (Fig. 4c), and that it is accompanied by alterations in bacterial metabolic pathway related to the production of SCFAs, which are known inducers of serotonin production [47]. We also show that the rise in serum serotonin was accompanied by an increased abundance of Sporanaerobacter of Clostridia class, a genus that forms spores and produces SCFAs [48]. This genus carries relevance in that a recent publication, through the use of germ-free mice, has skillfully demonstrated the role of spore-forming bacteria on the production of serotonin [10]. Thus, our results suggest that the widespread prevalence of hyperserotonemia in children with ASD might have a microbial basis.

In our study, it is possible that the metabolism and gut microbial status of the pre-weaned pups was largely affected by their mothers, possibly through milk ingestion. However, whereas Prevotellaceae was significantly reduced in the feces of offspring (Fig. 3a), the same phenomenon was not observed in the feces of their mothers (Additional file 2: Figure S2). Also, whereas serum from offspring right before weaning showed significantly increased amount of serotonin (Fig. 4c), their mothers did not show any statistically significant increase in their serum serotonin at any time point (Additional file 3: Figure S3). These results argue against the possibility that the mothers were the primary target of poly I:C and VPA injection in terms of microbial dysbiosis and hyperserotonemia.

A few papers have argued that the gut microbial composition of children with ASD do not differ significantly from those of HCs or siblings [75, 76]. The apparent discordances among microbiota studies on ASD children might stem from differences in sampling and sequencing methods or cohort designation regarding autism severity and the presence of GI disorders. Nevertheless, mounting clinical evidence shows that children with ASD do suffer from various GI disorders and IBD [4, 61, 77], and since microbial dysbiosis accounts for a significant part 
of the etiology of those conditions [7], gut microbiota in ASD warrants further study with a focus on adjusting for the high degree of inter-individual variability.

Microbiota as a research area is complex and rapidly growing, and its apparent involvement in ASD calls for targeted investigation of its relationship with the pathogenesis of the disorder. Our present study using animal model shows that (1) prenatal exposure to environmental risk factors is able to alter the abundances of many gut microbial taxa later in life, and that (2) such dysbiosis carries the possibility of directly affecting the host's health status via metabolic changes and serotonin pathway. Future research might benefit from focusing on the role of Prevotella in ASD and IBD, or describing in detail how ASD and IBD cause significant decreases in its abundance. We also suggest that poly I:C and VPA mouse are promising models for studying microbial dysbiosis in ASD, which might aid in developing a therapeutic regimen for ASD and/or IBD based on microbial manipulation.

\section{Additional files}

Additional file 1: Figure S1. ASD-related behavioral abnormalities in ASD mice. (a) Representative movement trajectories of CTL and ASD mice in 10-min sessions. Black square denotes the center area measured for exploratory behavior. (b) Comparison of total distance moved $(\mathrm{cm})$ in $\mathrm{CTL}$ and ASD mice. (c) Comparison of time spent in the center (black box) (s) in CTL and ASD mice. ${ }^{*} p<0.05$ vs. CTL. (d) Representative movement trajectories of CTL and ASD mice in a 3-chamber apparatus in 10-min sessions. The upper row shows experimental session testing for preference for the social cue (S1; stranger 1) over non-social cue (Ob; object) preference, and lower row shows the session testing for preference for the novel social cue (S2; stranger 2) over familiar social cue (S1; stranger 1). (e and f) Bar graph representation of time spent at stranger 1 over an object (e) and time spent at stranger 2 over stranger 1 (f). ${ }^{*} p<0.05$ vs. CTL. $n=7-10$ in all experiments. (PDF $578 \mathrm{~kb}$ )

Additional file 2: Figure S2. Relative abundance of Prevotellaceae in mother mice. n.s. $=$ not significant. $n=10-12$. (PDF $81 \mathrm{~kb}$ )

Additional file 3: Figure S3. Serum serotonin level in mother mice. n.s. $=$ not significant. $n=5$ each. (PDF $192 \mathrm{~kb}$ )

Additional file 4: Table S1. List of microbial taxa significantly altered in poly I:C and VPA mice. Only the taxa with more than $20 \%$ changes in abundance relative to CTL are shown. (XLSX $15 \mathrm{~kb}$ )

Additional file 5: Table S2. List of metabolic pathways significantly altered in ASD mice. Only the pathways with more than $5 \%$ changes relative to $C T L$ are shown. (XLSX $14 \mathrm{~kb}$ )

\section{Acknowledgements}

Not applicable.

\section{Funding}

This work was supported by the National Research Foundation of Korea NRF-2015R1A2A1A10054078 and NRF-2015M3C9A4053391.

\section{Availability of data and materials}

The sequences from this study are deposited in the European Nucleotide Archive under the study accession number PRJEB14629.

\section{Authors' contributions}

JSL, MYL, and GK conceived and designed the study. JSL, MYL, and CYB performed the experiments and analyzed the data. JSL, MYL, and GK wrote the manuscript. All authors read and approved the final manuscript.

\section{Competing interests}

The authors declare that they have no competing interests.

\section{Consent for publication}

Not applicable.

\section{Ethics approval and consent to participate}

All animal experiments were approved by the Seoul National University Institutional Animal Care and Use Committees and were conducted in accordance with the Guide for the Care and Use of Laboratory Animal (SNU-150406-2).

\section{Publisher's Note}

Springer Nature remains neutral with regard to jurisdictional claims in published maps and institutional affiliations.

\section{Author details}

${ }^{1}$ Department of Environmental Health Sciences, Graduate School of Public Health, Seoul National University, Seoul, Republic of Korea. ${ }^{2}$ Institute of Health and Environment, Seoul National University, Seoul, Republic of Korea. ${ }^{3}$ Research Group of Gut Microbiome, Korea Food Research Institute, Seongnam, Gyeonggi-do, Republic of Korea. ${ }^{4}$ Center for Human and Environmental Microbiome, Seoul National University, Seoul, Republic of Korea. ${ }^{5} \mathrm{~N}$-Bio, Seoul National University, Seoul, Republic of Korea. ${ }^{6}$ KoBioLabs, Inc., 1-Gwanak-ro, Gwanak-gu, Bldg 220, Rm 630, Seoul 151-746, Republic of Korea

Received: 24 November 2016 Accepted: 4 April 2017

Published online: 20 April 2017

\section{References}

1. Harrington RA, Lee L-C, Crum RM, Zimmerman AW, Hertz-Picciotto I. Serotonin hypothesis of autism: implications for selective serotonin reuptake inhibitor use during pregnancy. Autism Res. 2013;6:149-68.

2. Pagan C, Delorme R, Callebert J, Goubran-Botros H, Amsellem F, Drouot $X$, et al. The serotonin-N-acetylserotonin-melatonin pathway as a biomarker for autism spectrum disorders. Transl Psychiatry. 2014;4:e479.

3. Cook EH, Leventhal BL. The serotonin system in autism. Curr Opin Pediatr. 1996;8:348-54.

4. McElhanon BO, McCracken C, Karpen S, Sharp WG. Gastrointestinal symptoms in autism spectrum disorder: a meta-analysis. Pediatrics. 2014;133:872-83.

5. Doshi-Velez F, Avillach P, Palmer N, Bousvaros A, Ge Y, Fox K, et al. Prevalence of Inflammatory Bowel Disease Among Patients with Autism Spectrum Disorders. Inflamm Bowel Dis. 2015;21:2281-8.

6. Clemente JC, Ursell LK, Parfrey LW, Knight R. The impact of the gut microbiota on human health: An integrative view. Cell. 2012;148:1258-70.

7. Dupont AW, Dupont HL. The intestinal microbiota and chronic disorders of the gut. Nat Rev Gastroenterol Hepatol. 2011;8:523-31.

8. Cryan JF, Dinan TG. Mind-altering microorganisms: the impact of the gut microbiota on brain and behaviour. Nat Rev Neurosci. 2012;13:701-12.

9. Hsiao EY, McBride SW, Hsien S, Sharon G, Hyde ER, McCue T, et al. Microbiota Modulate Behavioral and Physiological Abnormalities Associated with Neurodevelopmental Disorders. Cell. 2013;155:1451-63.

10. Yano JM, Yu K, Donaldson GP, Shastri GG, Ann P, Ma L, et al. Indigenous bacteria from the gut microbiota regulate host serotonin biosynthesis. Cell. 2015;161:264-76.

11. Boyer EW, Shannon M. The serotonin syndrome. N Engl J Med. 2005;352:1112-20.

12. De Angelis M, Piccolo M, Vannini L, Siragusa S, De Giacomo A, Serrazzanetti DI, et al. Fecal Microbiota and Metabolome of Children with Autism and Pervasive Developmental Disorder Not Otherwise Specified. PLoS One. 2013;8:1-18.

13. Kang D-W, Park JG, Ihan ZE, Wallstrom G, LaBaer J, Adams JB, et al. Reduced Incidence of Prevotella and Other Fermenters in Intestinal Microflora of Autistic Children. PLoS One. 2013;8:e68322.

14. Sartor RB, Mazmanian SK. Intestinal Microbes in Inflammatory Bowel Diseases. Am J Gastroenterol Suppl. 2012;1:15-21. 
15. Willing BP, Dicksved J, Halfvarson J, Andersson AF, Lucio M, Zheng Z, et al. A pyrosequencing study in twins shows that gastrointestinal microbial profiles vary with inflammatory bowel disease phenotypes. Gastroenterology. 2010;139:1844-54. e1.

16. Elsabbagh M, Divan G, Koh Y-J, Kim YS, Kauchali S, Marcín C, et al. Global prevalence of autism and other pervasive developmental disorders. Autism Res. 2012;5:160-79.

17. Caporaso JG, Lauber CL, Walters WA, Berg-Lyons D, Huntley J, Fierer N, et al. Ultra-high-throughput microbial community analysis on the Illumina HiSeq and MiSeq platforms. ISME J. 2012;6:1621-4.

18. Lim MY, You HJ, Yoon HS, et al. The effect of heritability and host genetics on the gut microbiota and metabolic syndrome. Gut Published Online First: 06 April 2016. doi:10.1136/gutjnl-2015-311326.

19. Shannon P, Markiel A, Ozier O, Baliga NS, Wang JT, Ramage D, et al. Cytoscape: A software Environment for integrated models of biomolecular interaction networks. Genome Res. 2003;13:2498-504.

20. Lim MY, Yoon HS, Rho M, Sung J, Song Y-M, Lee K, et al. Analysis of the association between host genetics, smoking, and sputum microbiota in healthy humans. Sci Rep. 2016:6:23745.

21. Langille MGl, Zaneveld J, Caporaso JG, McDonald D, Knights D, Reyes JA et al. Predictive functional profiling of microbial communities using 165 rRNA marker gene sequences. Nat Biotechnol. 2013;31:814-21.

22. Lim MY, Rho M, Song Y-M, Lee K, Sung J, Ko G. Stability of gut enterotypes in Korean monozygotic twins and their association with biomarkers and diet. Sci Rep. 2014;4:7348.

23. Development R. Core Team RFFSC. R: A Language and Environment for Statistical Computing. Vienna Austria R Found. Stat Comput. 2008;1. 3-900051-07-0.

24. Hsiao EY, McBride SW, Chow J, Mazmanian SK, Patterson PH. Modeling an autism risk factor in mice leads to permanent immune dysregulation. Proc Natl Acad Sci U S A. 2012;109:12776-81.

25. Kataoka S, Takuma K, Hara Y, Maeda Y, Ago Y, Matsuda T. Autism-like behaviours with transient histone hyperacetylation in mice treated prenatally with valproic acid. Int J Neuropsychopharmacol. 2013;16(1):91-103.

26. Consortium THMP. Structure, Function and Diversity of the Healthy Human Microbiome. Nature. 2013;486:207-14

27. Finegold SM, Dowd SE, Gontcharova V, Liu C, Henley KE, Wolcott RD, et al. Pyrosequencing study of fecal microflora of autistic and control children. Anaerobe. 2010;16:444-53.

28. Finegold SM, Downes J, Summanen PH. Microbiology of regressive autism Anaerobe. 2012:18:260-2

29. Finegold SM. Desulfovibrio species are potentially important in regressive autism. Med Hypotheses. 2011;77:270-4.

30. Baron EJ. Bilophila wadsworthia: a unique Gram-negative anaerobic rod. Anaerobe. 1997;3:83-6.

31. Juste C, Kreil DP, Beauvallet C, Guillot A, Vaca S, Carapito C, et al. Bacterial protein signals are associated with Crohn's disease. Gut. 2014;63:1566-77.

32. Golińska E, Tomusiak A, Gosiewski T, Więcek G, Machul A, Mikołajczyk D, et al. Virulence factors of Enterococcus strains isolated from patients with inflammatory bowel disease. World J Gastroenterol. 2013;19:3562-72.

33. Rigsbee L, Agans R, Shankar V, Kenche H, Khamis HJ, Michail S, et al. Quantitative profiling of gut microbiota of children with diarrhea-predominant irritable bowel syndrome. Am J Gastroenterol. 2012;107:1740-51.

34. Saitoh S, Noda S, Aiba Y, Takagi A, Sakamoto M, Benno Y, et al. Bacteroides ovatus as the predominant commensal intestinal microbe causing a systemic antibody response in inflammatory bowel disease. Clin Diagn Lab Immunol. 2002;9:54-9.

35. Lavelle A, Lennon G, O'Sullivan O, Docherty N, Balfe A, Maguire A, et al. Spatial variation of the colonic microbiota in patients with ulcerative colitis and control volunteers. Gut. 2015;64:1553-61.

36. Gevers D, Kugathasan S, Denson LA, Vázquez-Baeza Y, Van Treuren W, Ren $B$, et al. The treatment-naive microbiome in new-onset Crohn's disease. Cell Host Microbe. 2014;15:382-92.

37. Rowan F, Docherty NG, Murphy M, Murphy B, Coffey JC, O'Connell PR. Desulfovibrio Bacterial Species Are Increased in Ulcerative Colitis. Dis Colon Rectum. 2010;53:1530-6.

38. Kaakoush NO, Day AS, Huinao KD, Leach ST, Lemberg DA, Dowd SE, et al. Microbial dysbiosis in pediatric patients with Crohn's disease. J Clin Microbiol. 2012;50:3258-66.

39. Jia W, Whitehead RN, Griffiths L, Dawson C, Waring RH, Ramsden DB, et al. Is the abundance of Faecalibacterium prausnitzii relevant to Crohn's disease? FEMS Microbiol Lett. 2010;310:138-44.
40. Balish E, Warner T. Enterococcus faecalis induces inflammatory bowel disease in interleukin-10 knockout mice. Am J Pathol. 2002;160:2253-7.

41. 41 Nishijo $M$, Pham $T$, Nguyen a TN, Tran NN, Nakagawa $H$, Hoang L V et al. 2,3,7,8-Tetrachlorodibenzo-p-dioxin in breast milk increases autistic traits of 3-year-old children in Vietnam. Mol Psychiatry 2014; : 1-7.

42. Baron-Cohen S, Auyeung B, Nørgaard-Pedersen B, Hougaard DM, Abdallah MW, Melgaard L, et al. Elevated fetal steroidogenic activity in autism. Mol Psychiatry. 2015;20:369-76.

43. 43 Carbonero F, Benefiel AC, Alizadeh-Ghamsari AH, Gaskins HR. Microbial pathways in colonic sulfur metabolism and links with health and disease. Front Physiol 2012; 3 NOV. doi:10.3389/fphys.2012.00448.

44. Theodoratou E, Campbell H, Ventham NT, Kolarich D, Pučić-Baković M, Zoldoš V, et al. The role of glycosylation in IBD. Nat Rev Gastroenterol Hepatol. 2014;11:588-600.

45. Emanuele E, Orsi P, Boso M, Broglia D, Brondino N, Barale F, et al. Low-grade endotoxemia in patients with severe autism. Neurosci Lett. 2010;471:162-5.

46. Rojo ÓP, San Román AL, Arbizu EA, Martínez ADLH, Sevillano ER, Martínez AA. Serum lipopolysaccharide-binding protein in endotoxemic patients with inflammatory bowel disease. Inflamm Bowel Dis. 2007;13:269-77.

47. Reigstad CS, Salmonson CE, Rainey JF, Szurszewski JH, Linden DR, Sonnenburg JL, et al. Gut microbes promote colonic serotonin production through an effect of short-chain fatty acids on enterochromaffin cells. FASEB J. 2015;29:1395-403.

48. Hernandez-Eugenio G, Fardeau ML, Cayol JL, Patel BKC, Thomas P, Macarie H, et al. Sporanaerobacter acetigenes gen. nov., sp. nov., a novel acetogenic, facultatively sulfur-reducing bacterium. Int J Syst Evol Microbiol. 2002;52:1217-23.

49. Adams JB, Johansen LJ, Powell LD, Quig D, Rubin R a. Gastrointestinal flora and gastrointestinal status in children with autism-comparisons to typical children and correlation with autism severity. BMC Gastroenterol. 2011;11:22.

50. Maukonen J, Kolho K-L, Paasela M, Honkanen J, Klemetti P, Vaarala O, et al. Altered Fecal Microbiota in Paediatric Inflammatory Bowel Disease. J Crohn's Colitis. 2015:9(12):1088-95.

51. Rooks MG, Veiga P, Wardwell-scott LH, Tickle T, Segata N, Michaud M, et al. Gut microbiome composition and function in experimental colitis during active disease and treatment-induced remission. ISME J. 2014;8: 1403-17.

52. Laukoetter MG, Nava P, Nusrat A. Role of the intestinal barrier in inflammatory bowel disease. World J Gastroenterol. 2008;14:401-7.

53. Arsenault D, St-Amour I, Cisbani G, Rousseau LS, Cicchetti F. The different effects of LPS and poly I: C prenatal immune challenges on the behavior, development and inflammatory responses in pregnant mice and their offspring. Brain Behav Immun. 2014;38:77-90.

54. De Theije CGM, Koelink PJ, Korte-Bouws GAH, Lopes da Silva S, Korte SM, Olivier B, et al. Intestinal inflammation in a murine model of autism spectrum disorders. Brain Behav Immun. 2014;37:240-7.

55. Koelink PJ, Overbeek SA, Braber S, Morgan ME, Henricks PAJ, Abdul Roda M et al. Collagen degradation and neutrophilic infiltration: a vicious circle in inflammatory bowel disease. Gut. 2014;63:578-87.

56. Roda G, Sartini A, Zambon E, Calafiore A, Marocchi M, Caponi A, et al. Intestinal epithelial cells in inflammatory bowel diseases. World J Gastroenterol. 2010;16:4264-71.

57. Kim J-W, Choi CS, Kim KC, Park JH, Seung H, Joo SH, et al. Gastrointestinal tract abnormalities induced by prenatal valproic Acid exposure in rat offspring. Toxicol Res. 2013;29:173-9.

58. De Filippo C, Cavalieri D, Di Paola M, Ramazzotti M, Poullet JB, Massart S, et al. Impact of diet in shaping gut microbiota revealed by a comparative study in children from Europe and rural Africa. Proc Natl Acad Sci U S A. 2010;107:14691-6.

59. Smith M, Flodman PL, Gargus JJ, Simon MT, Verrell K, Haas R, et al. Mitochondrial and ion channel gene alterations in autism. Biochim Biophys Acta. 1817;2012:1796-802.

60. Thomas Curtis J, Chen Y, Buck DJ, Davis RL. Chronic inorganic mercury exposure induces sex-specific changes in central TNF?? expression: Importance in autism? Neurosci Lett. 2011:504:40-4.

61. Coury DL, Ashwood P, Fasano A, Fuchs G, Geraghty M, Kaul A, et al. Gastrointestinal conditions in children with autism spectrum disorder: developing a research agenda. Pediatrics. 2012;130(Suppl):S160-8.

62. Nouel D, Burt M, Zhang Y, Harvey L, Boksa P. Prenatal exposure to bacterial endotoxin reduces the number of GAD67- and reelin-immunoreactive neurons in the hippocampus of rat offspring. Eur Neuropsychopharmacol. 2012;22:300-7. 
63. McCanlies EC, Fekedulegn D, Mnatsakanova A, Burchfiel CM, Sanderson WT, Charles LE, et al. Parental occupational exposures and autism spectrum disorder. J Autism Dev Disord. 2012;42:2323-34.

64. Adamsen D, Ramaekers V, Ho HT, Britschgi C, Rüfenacht V, Meili D, et al. Autism spectrum disorder associated with low serotonin in CSF and mutations in the SLC29A4 plasma membrane monoamine transporter (PMAT) gene. Mol Autism. 2014;5:43

65. Gu F, Chauhan V, Kaur K, Brown WT, LaFauci G, Wegiel J, et al. Alterations in mitochondrial DNA copy number and the activities of electron transport chain complexes and pyruvate dehydrogenase in the frontal cortex from subjects with autism. Transl Psychiatry. 2013;3:e299.

66. Pasternak BA, D'Mello S, Jurickova II, Han X, Willson T, Flick L, et al. Lipopolysaccharide exposure is linked to activation of the acute phase response and growth failure in pediatric Crohn's disease and murine colitis. Inflamm Bowel Dis. 2010;16:856-69.

67. Salvatore S, Heuschkel R, Tomlin S, Davies SE, Edwards S, Walker-Smith JA, et al. A pilot study of $\mathrm{N}$-acetyl glucosamine, a nutritional substrate for glycosaminoglycan synthesis, in paediatric chronic inflammatory bowel disease. Aliment Pharmacol Ther. 2000;14:1567-79.

68. Medicherla K, Sahu BD, Kuncha M, Kumar JM, Sudhakar G, Sistla R. Oral administration of geraniol ameliorates acute experimental murine colitis by inhibiting pro-inflammatory cytokines and NF-kB signaling. Food Funct. 2015;6:2984-95.

69. Fan F, Mundra PA, Fang L, Galvin A, Moore XL, Weir JM, et al. Lipidomic Profiling in Inflammatory Bowel Disease: Comparison Between Ulcerative Colitis and Crohn's Disease. Inflamm Bowel Dis. 2015;21:1511-8.

70. Kirsten TB, Palermo-Neto J, Bernardi MM. 11. A rat model of autism induced by a single early prenatal exposure to LPS. Brain Behav Immun. 2012;26:S4.

71. Foley KA, Ossenkopp KP, Kavaliers M, MacFabe DF. Pre- and neonatal exposure to lipopolysaccharide or the enteric metabolite, propionic acid alters development and behavior in adolescent rats in a sexually dimorphic manner. PLoS One 2014; 9. doi:10.1371/journal.pone.0087072

72. Schain RJ, Freedman DX. Studies on 5-hydroxyindole metabolism in autistic and other mentally retarded children. J Pediatr. 1961;58:315-20.

73. Veenstra-VanderWeele J, Muller CL, Iwamoto H, Sauer JE, Owens WA, Shah $\mathrm{CR}$, et al. Autism gene variant causes hyperserotonemia, serotonin receptor hypersensitivity, social impairment and repetitive behavior. Proc Natl Acad Sci U S A. 2012;109:5469-74.

74. Abrahams BS, Geschwind DH. Advances in autism genetics: on the threshold of a new neurobiology. Nat Rev Genet. 2008;9:341-55.

75. Son JS, Zheng LJ, Rowehl LM, Tian X, Zhang Y, Zhu W, et al. Comparison of fecal microbiota in children with autism spectrum disorders and neurotypical siblings in the simons simplex collection. PLoS One 2015; 10. doi:10.1371/journal.pone.0137725.

76. Tomova A, Husarova V, Lakatosova S, Bakos J, Vlkova B, Babinska K, et al. Gastrointestinal microbiota in children with autism in Slovakia. Physiol Behav. 2015;138:179-87.

77. Chaidez V, Hansen RL, Hertz-Picciotto I. Gastrointestinal problems in children with autism, developmental delays or typical development. J Autism Dev Disord. 2014:44:1117-27.

78. Zitomersky NL, Atkinson BJ, Franklin SW, Mitchell PD, Snapper SB, Comstock LE et al. Characterization of Adherent Bacteroidales from Intestinal Biopsies of Children and Young Adults with Inflammatory Bowel Disease. PLoS One 2013; 8. doi:10.1371/journal.pone.0063686.

79. Sokol H, Seksik P, Furet JP, Firmesse O, Nion-Larmurier I, Beaugerie L, et al. Low counts of faecalibacterium prausnitzii in colitis microbiota. Inflamm Bowel Dis. 2009;15:1183-9.

\section{Submit your next manuscript to BioMed Central and we will help you at every step:}

- We accept pre-submission inquiries

- Our selector tool helps you to find the most relevant journal

- We provide round the clock customer support

- Convenient online submission

- Thorough peer review

- Inclusion in PubMed and all major indexing services

- Maximum visibility for your research

Submit your manuscript at www.biomedcentral.com/submit
Biomed Central 